\title{
Short-Term Fasting Reduces the Extent of Myocardial Infarction and Incidence of Reperfusion Arrhythmias in Rats
}

\author{
M. ŠNOREK ${ }^{1,3,5}$, D. HODYC ${ }^{1,5}$, V. ŠEDIVÝ $Y^{1,5}$, J. ĎURIŠOVÁ ${ }^{5}$, A. SKOUMALOVÁ ${ }^{2,5}$, \\ J. WILHELM ${ }^{2,5}$, J. NECKÁR ${ }^{4,5}$, F. KOLÁŘR ${ }^{4,5}$, J. HERGET $^{1,5}$
}

${ }^{1}$ Department of Physiology, Second Faculty of Medicine, Charles University in Prague, Prague, Czech Republic, ${ }^{2}$ Department of the Medical Chemistry and Biochemistry, Second Faculty of Medicine, Charles University in Prague, Prague, Czech Republic, ${ }^{3}$ Heart Center, České Budějovice Hospital, České Budějovice, Czech Republic, ${ }^{4}$ Institute of Physiology, Academy of Sciences of the Czech Republic, Prague, Czech Republic, ${ }^{5}$ Centre for Cardiovascular Research, Prague, Czech Republic

Received February 16, 2012

Accepted July 23, 2012

On-line October 25, 2012

\section{Summary}

The effect of three-day fasting on cardiac ischemic tolerance was investigated in adult male Wistar rats. Anesthetized open-chest animals (pentobarbitone $60 \mathrm{mg} / \mathrm{kg}$, i.p.) were subjected to 20-min left anterior descending coronary artery occlusion and 3-h reperfusion for infarct size determination. Ventricular arrhythmias were monitored during ischemia and at the beginning ( $3 \mathrm{~min}$ ) of reperfusion. Myocardial concentrations of beta-hydroxybutyrate and acetoacetate were measured to assess mitochondrial redox state. Short-term fasting limited the infarct size $(48.5 \pm 3.3 \%$ of the area at risk) compared to controls $(74.3 \pm 2.2 \%)$ and reduced the total number of premature ventricular complexes $(12.5 \pm 5.8)$ compared to controls $(194.9 \pm 21.9)$ as well as the duration of ventricular tachycardia $(0.6 \pm 0.4 \mathrm{~s}$ vs. $18.8 \pm 2.5 \mathrm{~s})$ occurring at early reperfusion. Additionally, fasting increased the concentration of beta-hydroxybutyrate and betahydroxybutyrate/acetoacetate ratio $(87.8 \pm 27.0)$ compared to controls $(7.9 \pm 1.7)$, reflecting altered mitochondrial redox state. It is concluded that three-day fasting effectively protected rat hearts against major endpoints of acute I/R injury. Further studies are needed to find out whether these beneficial effects can be linked to altered mitochondrial redox state resulting from increased ketogenesis.

\section{Key words}

Myocardial ischemia/reperfusion - Arrhythmias - Infarction • Fasting • Ketone bodies

\section{Corresponding author}

M. Šnorek, Department of Physiology, Second Faculty of Medicine, Charles University in Prague, Plzeňská 130/221, 15006 Prague 5, Czech Republic. Fax: +420-257-210-995. E-mail: michal.snorek@Ifmotol.cuni.cz

\section{Introduction}

After an acute myocardial infarction, early myocardial reperfusion is the only effective strategy for reducing the extent of infarct size and improving the clinical outcome. However, restoring the blood flow to the ischemic myocardium may induce myocardial reperfusion injury, which reduces the beneficial effects of reperfusion (Yellon and Hausenloy 2007). It is now established that reactive oxygen species (ROS) play a role in the pathogenesis of myocardial injury associated especially with reperfusion. It has been well documented that ROS contribute to early reperfusion arrhythmias and that antioxidants can significantly attenuate their incidence and severity (reviewed in Li and Jackson 2002, Zweier and Talukder 2006). In contrast to the involvement of ROS in reperfusion arrhythmias, it is still debated whether ROS significantly contribute to other end points of ischemia/reperfusion ( $\mathrm{I} / \mathrm{R})$ injury. Although numerous experimental studies demonstrated beneficial effects of ROS dampening on ischemic arrhythmias and infarct size, other reports showed only minor (if any) protection (e.g. 
Miura et al. 1988, Neckar et al. 2008, Imani et al. 2011). Nevertheless, prevention of production of cytotoxic ROS might be a target for effective cardioprotection, especially during the reperfusion phase.

It has been shown that cell redox state correlates with ROS formation (Stowe and Camara 2009, Wolin 2009, Aon et al. 2010). Formation of ROS and subsequent oxidative damage can be reduced by various regimens of long-term dietary restrictions such as reduced energy intake (Gredilla et al. 2001, Bevilacqua et al. 2004, Colom et al. 2007) or intermittent fasting (Wan et al. 2003, Johnson et al. 2007). Caloric restriction is related to the concept of so called reductive stress. Under normoxic conditions mitochondria operate in a physiological intermediate redox state (Aon et al. 2010). The redox couples comprise the relatively oxidized $\mathrm{NAD}^{+} / \mathrm{NADH}$, and relatively reduced couples $\mathrm{NADP}^{+} / \mathrm{NADPH}$ and GSSG/GSH. The formation of ROS is supported primarily by reduced pyridine nucleotides, such as NADH or NADPH (Shen et al. 2005). Thus, under the conditions of increased concentration of reduced nucleotides, higher rates of ROS production can occur. The increase of reductive power in the mitochondrial compartment is reflected by the increased beta-hydroxybutyrate/acetoacetate ratio (Williamson et al. 1967). Beta-hydroxybutyrate (i.e. 3-hydroxybutyrate, $\mathrm{BHB}$ ) and acetoacetate (AcAc) represent two main ketone bodies (Mitchell et al. 1995). The reaction of AcAc with NADH and hydrogen ion, catalyzed by betahydroxybutyrate dehydrogenase (Hegardt 1999), results in the formation of BHB and $\mathrm{NAD}^{+}$. Fasting of laboratory animals increases ketogenesis (Mitchell et al. 1995), which can affect cell redox state and ROS formation by consuming reduced nucleotides.

While cardioprotective effects of various regimens of long-term dietary restrictions have been well documented (e.g. Chandrasekar et al. 2001, Ahmet et al. 2005, Varady et al. 2009), the impact of short-term fasting (days before the ischemic insult) on cardiac tolerance to acute $\mathrm{I} / \mathrm{R}$ injury has been rarely examined. In the present study, we therefore evaluated infarct size and the incidence of ventricular arrhythmias induced by regional $\mathrm{I} / \mathrm{R}$ in rats subjected to fasting for 3 days. Redox state of myocardial mitochondria was assessed by measuring the $\mathrm{BHB} / \mathrm{AcAc}$ ratio.

\section{Methods}

Adult male Wistar rats (AnLab, Czech Republic) were divided into two groups. Animals in the experimental group did not have access to food for 3 days but had free access to common drinking water from supply system. The duration of fasting was determined arbitrarily. Control group had free access to water and a standard laboratory diet. All experiments were performed in accordance with the European Community and NIH guidelines for use of experimental animals and approved by the animal studies committee at our institution.

\section{Surgical procedure}

Animals were anesthetized by sodium pentobarbitone (Sanofi, France; $60 \mathrm{mg} / \mathrm{kg}$ body weight, i.p.). Heparinized cannula was placed in the left carotid artery for blood pressure monitoring with a pressure transducer (Gould P23Gb, USA). Tracheotomy was performed, the rats were intubated with a cannula connected to a rodent ventilator (Ugo Basile, Italy) and ventilated with room air at $65-70$ breaths/min (tidal volume of $1.2 \mathrm{ml} / 100 \mathrm{~g}$ body weight). A single-lead electrocardiogram (ECG) was continually recorded. Both blood pressure and ECG signals were subsequently analyzed by our custom-designed software. The rectal temperature was maintained within 36.5 and $37.5^{\circ} \mathrm{C}$ by a heated table throughout the experiment. Left thoracotomy was performed and left anterior descending (LAD) coronary artery was occluded for 20 minutes about 1-2 $\mathrm{mm}$ distal to the origin. Characteristic changes in the configuration of the ECG and a transient decrease in blood pressure verified the coronary artery occlusion. After releasing the occlusion reperfusion was indicated by changes of the ECG, transient decrease of blood pressure and appearance of reperfusion arrhythmias.

\section{Analysis of arrhythmias}

The incidence and severity of ventricular arrhythmias during ischemic insult and the first $3 \mathrm{~min}$ of reperfusion were assessed according to the Lambeth Convention (Walker et al. 1988). Premature ventricular complexes (PVCs) occurring as singles, salvos or tachycardia (a run of 4 or more consecutive PVCs) were counted separately. The incidence of ventricular tachycardia (VT) and fibrillation (VF) was also evaluated. VF lasting more than two minutes was considered as sustained (VFs).

\section{Measurement of infarct size}

After 3 hours of reperfusion, the hearts were excised and washed with $20 \mathrm{ml}$ saline through the 
cannulated aorta. The area at risk and the infarct size were determined as described elsewhere (Neckar et al. 2002) by staining with potassium permanganate (Lachema, Czech Republic) and 2,3,5triphenyltetrazolium chloride (Sigma, USA) dissolved in $0.1 \mathrm{~mol} / \mathrm{l}$ phosphate buffer ( $\mathrm{pH} 7.4$ ). The hearts were cut perpendicularly to the long axis of the left ventricle into slices $1 \mathrm{~mm}$ thick and stored overnight in $10 \%$ neutral formaldehyde solution. The day after the heart staining, the right ventricular free wall was separated and both sides of slices were photographed. The infarct size (IS), the size of the area at risk (AR) and the size of the left ventricle (LV) were determined by a computerized planimetric method. The IS was normalized to the AR (IS/AR) and the size of AR was normalized to the LV $(\mathrm{AR} / \mathrm{LV})$.

\section{Redox state assessment}

In separate groups of anesthetized open-chest animals, the hearts were excised either before or after $20 \mathrm{~min}$ of myocardial ischemia, the atria and right ventricle were removed and the left ventricle was frozen in liquid nitrogen. About $1.5 \mathrm{~g}$ of pre-weighted, minced, frozen tissue was added to the same volume of perchloric acid and after 10 min of proteins precipitation the mixture was centrifuged $(6,000 \mathrm{RPM}, 30 \mathrm{~min})$. Then the supernatant was separated and neutralized by potassium carbonate. After $20 \mathrm{~min}$, it was centrifuged again for $15 \mathrm{~min}$ and the homogenized supernatant was divided into three aliquots (each about $0.5 \mathrm{~g}$ ) used for measurements. Concentrations of BHB and AcAc were assessed according to Mintz and Robin (Mintz and Robin 1971). Briefly, BHB was determined by adding betahydroxybutyrate dehydrogenase (Sigma, USA), NAD+ and tris buffer ( $\mathrm{pH} 8.5)$ to the final supernatant; NADH was measured using spectrophotometer He $\lambda$ ios (Unicam, United Kingdom). In a similar manner, concentration of AcAc was measured by adding NADH, tris buffer ( $\mathrm{pH}$ 7.0) and the appropriate enzyme to the final supernatant, and by measuring the decrease in fluorescence as the NADH was oxidized to NAD+.

\section{Statistical analysis}

All results are expressed as means \pm S.E.M. The statistical significance of differences between groups was determined by one-way ANOVA and subsequent Fisher's PLSD test and the Games/Howell post-hoc test, as appropriate. Differences were considered as statistically significant when $\mathrm{p}<0.05$.

\section{Results}

Ischemia/reperfusion arrhythmias and infarct size

Three-day fasting tended to reduce the incidence of ischemic ventricular arrhythmias as documented by total number of PVCs (Fig. 1A) and duration of ventricular tachycardia (Fig. 1B) by about $40-50 \%$ but the differences between fasted and control groups did not reach statistical significance. However, fasting resulted in a significant suppression of arrhythmias occurring at early reperfusion as indicated by marked decreases of both parameters (Fig. 2A,B).
A

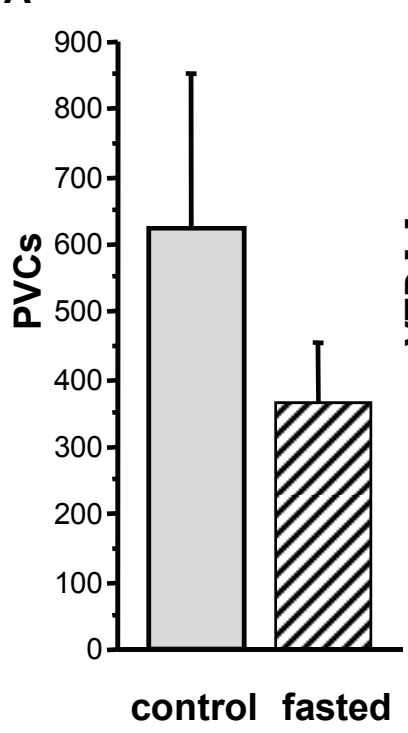

B

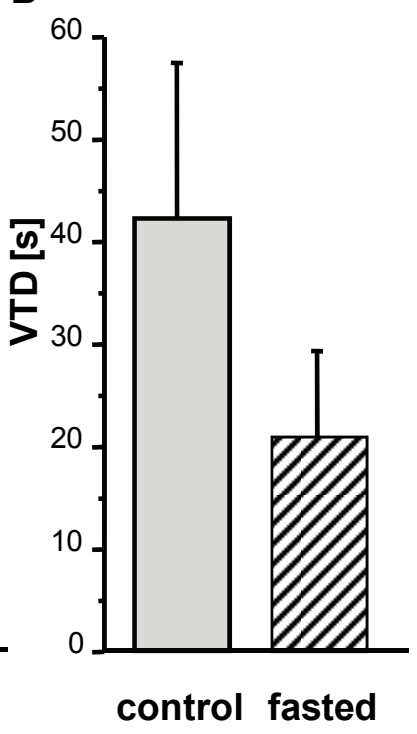

Fig. 1. (A) Total number of premature ventricular complexes (PVCs) and (B) total duration of ventricular tachycardia (VTD) over 20-min ischemic period in control and fasted rats. Values are means \pm S.E.M. from 12 fasted rats and 11 control animals.

The area at risk normalized to the left ventricle did not significantly differ between the fasted and control groups $(38.5 \pm 3.1 \%$ and $42.3 \pm 4.8 \%$, respectively). The infarct size in the control group reached $74.3 \pm 2.2 \%$ of the area at risk and it was significantly decreased by fasting to $48.5 \pm 3.3 \%$ (Fig. 3 ).

\section{Concentrations of ketone bodies}

Myocardial concentrations of ketone bodies are shown in Table 1. Fasting significantly increased myocardial concentration of BHB to $15.58 \pm 2.56 \mathrm{mM} / \mathrm{g}$ compared with control animals $(1.22 \pm 0.21 \mathrm{mM} / \mathrm{g})$ but the concentration of AcAc did not differ between the groups. Although the ratio of $\mathrm{BHB} / \mathrm{AcAc}$ increased six-fold in the fasted group, the difference did not reach statistical 
A

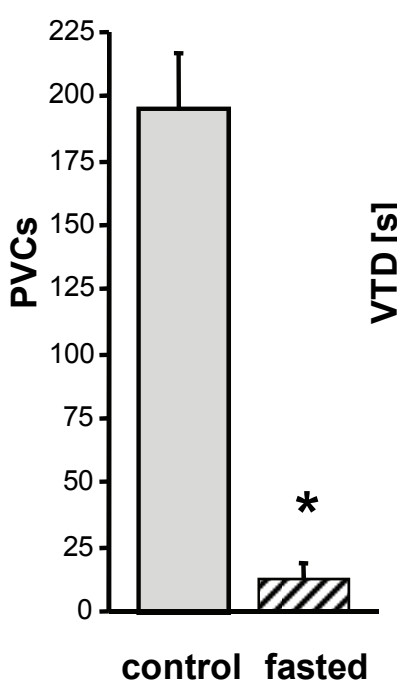

B

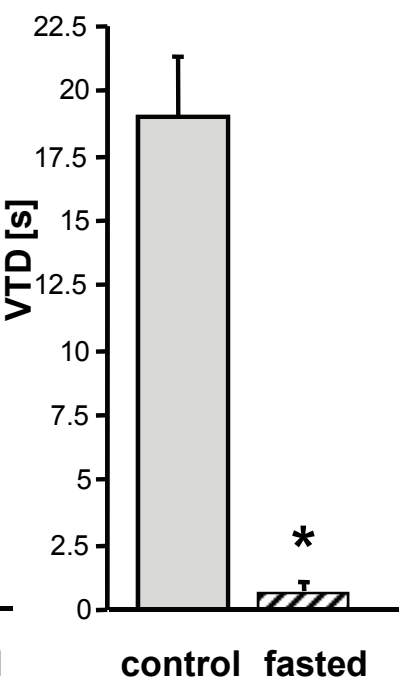

Fig. 2. (A) Total number of premature ventricular complexes (PVCs) and (B) total duration of ventricular tachycardia (VTD) during the first $3 \mathrm{~min}$ of reperfusion in control and fasted rats. Values are means \pm S.E.M. from 12 fasted rats and 11 control animals. $* P<0.05$.

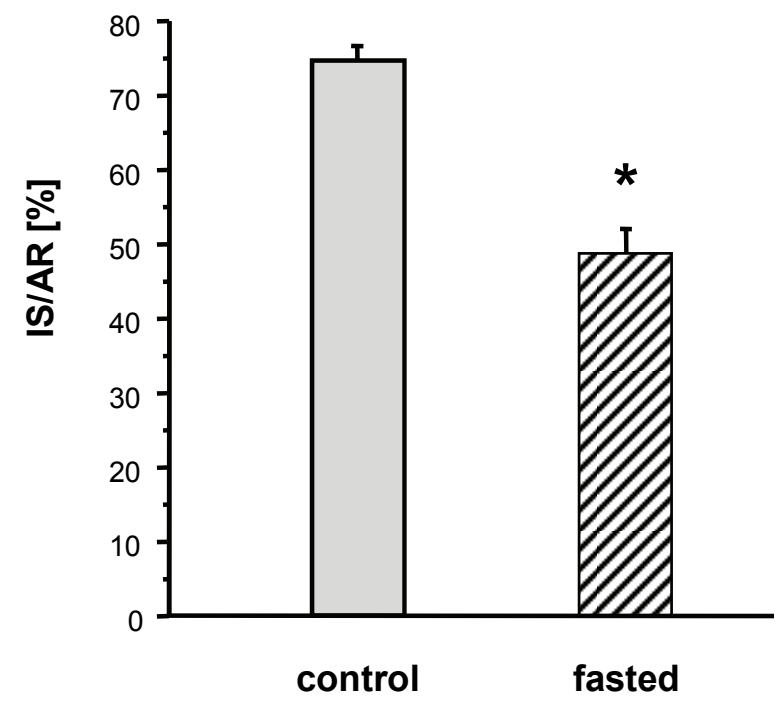

Fig. 3. Myocardial infarct size expressed as a percentage of the area at risk (IS/AR) in control and fasted rats. Values are means \pm S.E.M. from 6 fasted rats and 5 control animals. $* P<0.05$.

significance due to high variation in this group (Fig. 4). Ischemia tended to further increase the BHB concentration in the fasted group compared with controls while the concentration of AcAc remained unchanged in both groups. Consequently, the BHB/AcAc ratio after ischemia was significantly higher in the fasted group (Fig. 4).

\section{Discussion}

The present study demonstrated that short-term water-only fasting of rats limited the extent of acute

Table 1. Concentrations of beta-hydroxybutyrate (BHB) and acetoacetate (ACAC) in left ventricular myocardium of control and fasted rats before and after 20-min ischemia.

\begin{tabular}{lccc}
\hline Group & $\mathbf{n}$ & $\begin{array}{c}\text { BHB } \\
(\mathbf{m M} / \mathbf{g})\end{array}$ & $\begin{array}{c}\text { AcAc } \\
(\mathbf{m M} / \mathbf{g})\end{array}$ \\
\hline Control & 9 & $1.22 \pm 0.21$ & $0.29 \pm 0.07$ \\
Fasted & 9 & $15.58 \pm 2.56^{*}$ & $0.70 \pm 0.20$ \\
Control + ischemia & 9 & $2.24 \pm 0.35$ & $0.34 \pm 0.06$ \\
Fasted + ischemia & 11 & $18.71 \pm 3.15^{*}$ & $0.38 \pm 0.08$
\end{tabular}

Values are means \pm S.E.M. $* P<0.05$ vs. controls.

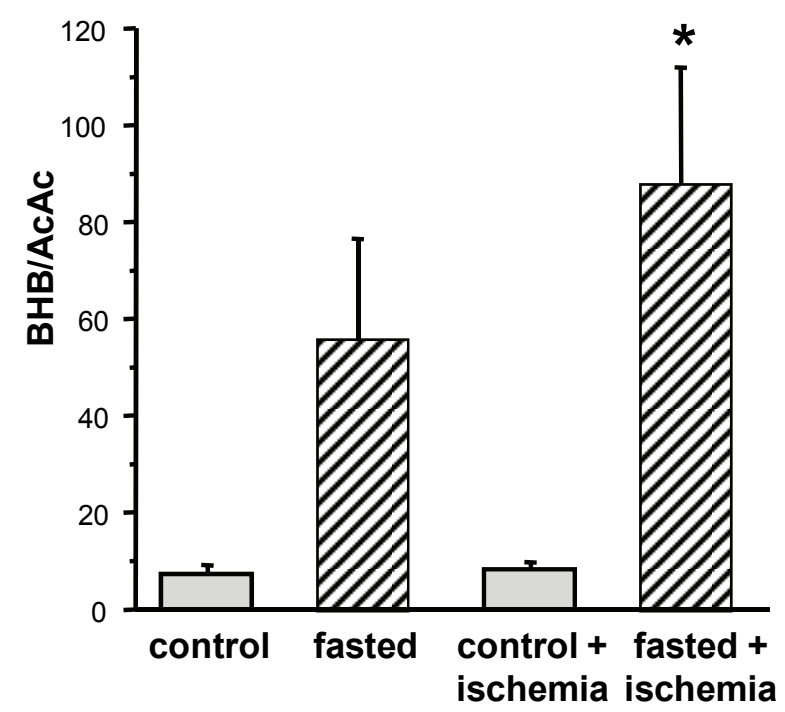

Fig. 4. Beta-hydroxybutyrate/acetoacetate (BHB/AcAc) ratio in left ventricular myocardium of control and fasted rats before and after 20-min ischemia. Values are means \pm S.E.M. from 9-11 hearts in each group. $* P<0.05$ vs. controls.

myocardial infarction induced by regional $\mathrm{I} / \mathrm{R}$ and markedly reduced the total number of PVCs and duration of ventricular tachycardia occurring at early reperfusion period. Additionally, fasting increased the myocardial $\mathrm{BHB} / \mathrm{AcAc}$ ratio reflecting altered mitochondrial redox state. Consistent with our observation, fasting of rats for only $24 \mathrm{~h}$ improved the postischemic recovery of contractile function and reduced the lactate dehydrogenase release in isolated hearts subjected to global I/R (Marina Prendes et al. 2005). Hearts of overnight (16-20 h) fasted rats also exhibited better recovery of contractile performance and preservation of the structural integrity of mitochondria following I/R than hearts of fed animals (Doenst et al. 1996). Moreover, 3 days of water-only fasting conferred protection against renal $\mathrm{I} / \mathrm{R}$ injury in mice, similar in magnitude to 2-4 weeks of $30 \%$ dietary restriction. Interestingly, the 
functional protection of kidneys by this short-term fast was rapidly lost within hours of refeeding (Mitchell et al. 2010) suggesting that the underlying mechanism(s) may differ from that of the long-term dietary restriction.

Although the cardioprotective mechanism of short-term fasting remains poorly understood, it seems likely that the decreased ROS formation and reduced tissue oxidative damage may play at least partial role. It was shown that hearts of rats fasted for $24 \mathrm{~h}$ exhibited higher GSH/GSSG ratio and lower levels of lipid peroxidation markers after $\mathrm{I} / \mathrm{R}$ than hearts of fed animals (Marina Prendes et al. 2009). There is ample evidence that longer durations of various dietary restriction regimens also limited formation of ROS. For example, Bevilacqua et al. (2004) showed decreased skeletal muscle mitochondrial production of hydrogen peroxide in rats subjected to $40 \%$ caloric restriction for 2 weeks to 6 months. Similar regimen (lasting 6 weeks) lowered hydrogen peroxide production in rat liver mitochondria (Gredilla et al. 2001). Judge et al. (2004) also reported lower myocardial mitochondrial hydrogen peroxide production in the calorie restricted rats (10-40\% restriction during 2 months) compared to fed animals. Furthermore, 3 months of $40 \%$ caloric restriction in rats resulted in lower content and improved efficiency of myocardial mitochondria that generated less hydrogen peroxide than fed animals, consistent with attenuated tissue oxidative damage (Colom et al. 2007). As recently demonstrated, 8 weeks of intermittent fasting in overweight humans markedly decreased markers of oxidative stress such as 8-isoprostane, nitrotyrosine and protein carbonyls (Johnson et al. 2007) supporting clinical relevance of the above-mentioned animal data.

Although neither ROS formation nor oxidative stress markers were measured in the present study, we found significant effect of three-day fasting on mitochondrial redox state revealed by assessing the $\mathrm{BHB} / \mathrm{AcAc}$ ratio. The increased production of ketone bodies and the BHB/AcAc was detected also after prolonged fasting (Laffel 1999). These data are in line with the concept of reductive stress which can increase ROS formation by several mechanisms including (1) mitochondrial leak due to nonenzymatic reactions, (2) increased activity of $\mathrm{NAD}(\mathrm{P}) \mathrm{H}$ oxidase, (3) increased activity of xanthine oxidase, (4) increased cyclooxygenase activity, and (5) reduction of ferric ions in ferritin to the ferrous state (Williamson et al. 1993, Tilton et al. 1997). However, this issue remains controversial (Ghyczy and Boros 2001, Lipinski 2002, Zhang et al.
2010) and further studies are needed to elucidate the role of ketone bodies in limiting ROS formation and oxidative injury. Nevertheless, our finding of altered mitochondrial redox state points to mitochondria as a key structure in the acquisition of cardiac tolerance to $\mathrm{I} / \mathrm{R}$ injury. In particular, mitochondrial ATP-sensitive potassium channels $\left(\mathrm{mK}_{\mathrm{ATP}}\right)$ and permeability transition pore (mPTP) are important ROS-regulated targets of various forms of cardioprotection. Limited evidence suggests that the protective mechanism of short-term fasting against I/R-induced myocardial contractile dysfunction involves both the activation of $\mathrm{mK}_{\mathrm{ATP}}$ (Marina Prendes et al. 2005) and prevention of MPTP opening (Marina Prendes et al. 2009). The potential role of these components in fastinginduced cardioprotection against other endpoints of $\mathrm{I} / \mathrm{R}$ injury is unknown. Recent studies in isolated heart model have tested activators of AMP-activated protein kinase (AMPK), a stress signaling enzyme involved in regulation of energy-generating and -consuming pathways, to protect the heart against ischemia. It was suggested that possible cardioprotective effect could be mediated through the inhibition of MPTP opening (Kim et al. 2011, Paiva et al. 2011).

It has been shown that administration of exogenous BHB reduced myocardial infarct size and apoptosis induced by $\mathrm{I} / \mathrm{R}$ in rats (Zou et al. 2002). Considering the role of ketone bodies in cardioprotection induced by fasting, the comparison with early stages of diabetes mellitus might be of interest. Chen et al. (1984) examining effects of experimental streptozotocin-induced diabetes in rats on myocardial metabolism demonstrated significant hyperketonemia as early as 2 days after the streptozotocin injection. Interestingly, smaller infarct size compared to that of non-diabetic controls was found in rats subjected to myocardial $\mathrm{I} / \mathrm{R}$ one week after the induction of diabetes (Ravingerova et al. 2003). Thus, it cannot be excluded that short-term fasting and early stages of experimental diabetes may utilize, at least in part, similar mechanisms to improve cardiac ischemic tolerance, possibly involving ketone bodies.

Short-term water-only fasting of animals is likely associated with stress which can precondition their hearts against subsequent stresses including I/R. In fact, cardiac protection induced by fasting was shown to be as effective as ischemic preconditioning (Doenst et al. 1996). In view of the complex molecular mechanism of preconditioning, it can be assumed that protective effects of fasting may also involve a variety of cell survival pathways. For example, it can be speculated that 
ischemia-tolerant phenotype of fasted animals is attributed to increased sympathetic stimulation and protective signaling triggered by catecholamines. The ability of catecholamines to precondition the heart against $\mathrm{I} / \mathrm{R}$ injury has been well documented (Ravingerova et al. 1995, Lochner et al. 1999).

In conclusion, our results demonstrated that three-days fasting effectively protected rat hearts against two major endpoints of acute $\mathrm{I} / \mathrm{R}$ injury: myocardial infarction and reperfusion ventricular arrhythmias. These beneficial effects can be linked to altered mitochondrial redox state resulting from increased ketogenesis but further studies are needed to test this possibility.

\section{Conflict of Interest}

There is no conflict of interest.

\section{Acknowledgements}

Supported by the Grant Agency of Charles University (60009/2009), Centre for Cardiovascular Research (MSMT 1M 0510) and Czech Science Foundation (GACR 305/08/0108).

\section{References}

AHMET I, WAN R, MATTSON MP, LAKATTA EG, TALAN M: Cardioprotection by intermittent fasting in rats. Circulation 112: 3115-3121, 2005.

AON MA, CORTASSA S, O'ROURKE B: Redox-optimized ROS balance: a unifying hypothesis. Biochim Biophys Acta 1797: 865-877, 2010.

BEVILACQUA L, RAMSEY JJ, HAGOPIAN K, WEINDRUCH R, HARPER ME: Effects of short- and medium-term calorie restriction on muscle mitochondrial proton leak and reactive oxygen species production. Am J Physiol Endocrinol Metab 286: E852-E861, 2004.

CHANDRASEKAR B, NELSON JF, COLSTON JT, FREEMAN GL: Calorie restriction attenuates inflammatory responses to myocardial ischemia-reperfusion injury. Am J Physiol Heart Circ Physiol 280: H2094-H2102, 2001.

CHEN V, IANUZZO CD, FONG BC, SPITZER JJ: The effects of acute and chronic diabetes on myocardial metabolism in rats. Diabetes 33: 1078-1084, 1984.

COLOM B, OLIVER J, ROCA P, GARCIA-PALMER FJ: Caloric restriction and gender modulate cardiac muscle mitochondrial $\mathrm{H}_{2} \mathrm{O}_{2}$ production and oxidative damage. Cardiovasc Res 74: 456-465, 2007.

DOENST T, GUTHRIE PH, CHEMNITIUS JM, ZECH R, TAEGTMEYER H: Fasting, lactate, and insulin improve ischemia tolerance in rat heart: a comparison with ischemic preconditioning. Am J Physiol 270: H1607-H1615, 1996.

GHYCZY M, BOROS M: Electrophilic methyl groups present in the diet ameliorate pathological states induced by reductive and oxidative stress: a hypothesis. Br J Nutr 85: 409-414, 2001.

GREDILLA R, BARJA G, LOPEZ-TORRES M: Effect of short-term caloric restriction on $\mathrm{H} 2 \mathrm{O} 2$ production and oxidative DNA damage in rat liver mitochondria and location of the free radical source. J Bioenerg Biomembr 33: 279-287, 2001.

HEGARDT FG: Mitochondrial 3-hydroxy-3-methylglutaryl-CoA synthase: a control enzyme in ketogenesis. Biochem J 338: 569-582, 1999.

IMANI A, FAGHIHI M, SADR SS, NIARAKI SS, ALIZADEH AM: Noradrenaline protects in vivo rat heart against infarction and ventricular arrhythmias via nitric oxide and reactive oxygen species. J Surg Res 169: 9-15, 2011.

JOHNSON JB, SUMMER W, CUTLER RG, MARTIN B, HYUN DH, DIXIT VD, PEARSON M, NASSAR M, TELLJOHANN R, MAUDSLEY S, CARLSON O, JOHN S, LAUB DR, MATTSON MP: Alternate day calorie restriction improves clinical findings and reduces markers of oxidative stress and inflammation in overweight adults with moderate asthma. Free Radic Biol Med 42: 665-674, 2007.

JUDGE S, JUDGE A, GRUNE T, LEEUWENBURGH C: Short-term CR decreases cardiac mitochondrial oxidant production but increases carbonyl content. Am J Physiol Regul Integr Comp Physiol 286: R254-R259, 2004. 
KIM AS, MILLER EJ, WRIGHT TM, LI J, QI D, ATSINA K, ZAHA V, SAKAMOTO K, YOUNG LH: A small molecule AMPK activator protects the heart against ischemia-reperfusion injury. J Mol Cell Cardiol 51: 24-32, 2011.

LAFFEL L: Ketone bodies: a review of physiology, pathophysiology and application of monitoring to diabetes. Diabetes Metab Res Rev 15: 412-426, 1999.

LI C, JACKSON RM: Reactive species mechanisms of cellular hypoxia-reoxygenation injury. Am J Physiol Cell Physiol 282: C227-C241, 2002.

LIPINSKI B: Evidence in support of a concept of reductive stress. Br J Nutr 87: 93-94; discussion 94, 2002.

LOCHNER A, GENADE S, TROMP E, PODZUWEIT T, MOOLMAN JA: Ischemic preconditioning and the betaadrenergic signal transduction pathway. Circulation 100: 958-966, 1999.

MARINA PRENDES MG, GARCIA JV, FERNANDEZ MA, PEREZ MJ, PERAZZO JC, SAVINO EA, VARELA A: Effects of 5-hydroxydecanoate and ischemic preconditioning on the ischemic-reperfused heart of fed and fasted rats. J Physiol Biochem 61: 447-456, 2005.

MARINA PRENDES MG, GONZALEZ MS, TORRESIN ME, HERMANN R, PASCALE NG, DEL MAR JAITOVICH M, SAVINO EA, VARELA A: Involvement of mitochondrial permeability transition, glutathione status, pentose phosphate pathway and oxidative damage in the protective effect of fasting on the ischaemicreperfused rat heart. Clin Exp Pharmacol Physiol 36: 637-642, 2009.

MINTZ S, ROBIN ED: Redox state of free nicotinamide-adenine nucleotides in the cytoplasm and mitochondria of alveolar macrophages. J Clin Invest 50: 1181-1186, 1971.

MITCHELL GA, KASSOVSKA-BRATINOVA S, BOUKAFTANE Y, ROBERT MF, WANG SP, ASHMARINA L, LAMBERT M, LAPIERRE P, POTIER E: Medical aspects of ketone body metabolism. Clin Invest Med 18: 193-216, 1995.

MITCHELL JR, VERWEIJ M, BRAND K, VAN DE VEN M, GOEMAERE N, VAN DEN ENGEL S, CHU T, FORRER F, MULLER C, DE JONG M, VAN IJCKEN W, IJZERMANS JN, HOEIJMAKERS JH, DE BRUIN RW: Short-term dietary restriction and fasting precondition against ischemia reperfusion injury in mice. Aging Cell 9: 40-53, 2010.

MIURA T, DOWNEY JM, HOTTA D, IIMURA O: Effect of superoxide dismutase plus catalase on myocardial infarct size in rabbits. Can J Cardiol 4: 407-411, 1988.

NECKAR J, PAPOUSEK F, NOVAKOVA O, OSTADAL B, KOLAR F: Cardioprotective effects of chronic hypoxia and ischaemic preconditioning are not additive. Basic Res Cardiol 97: 161-167, 2002.

NECKAR J, OSTADAL B, KOLAR F: Acute but not chronic tempol treatment increases ischemic and reperfusion ventricular arrhythmias in open-chest rats. Physiol Res 57: 653-656, 2008.

PAIVA MA, RUTTER-LOCHER Z, GONCALVES LM, PROVIDENCIA LA, DAVIDSON SM, YELLON DM, MOCANU MM: Enhancing AMPK activation during ischemia protects the diabetic heart against reperfusion injury. Am J Physiol Heart Circ Physiol 300: H2123-H2134, 2011.

RAVINGEROVA T, NECKAR J, KOLAR F: Ischemic tolerance of rat hearts in acute and chronic phases of experimental diabetes. Mol Cell Biochem 249: 167-174, 2003.

RAVINGEROVA T, PYNE NJ, PARRATT JR: Ischaemic preconditioning in the rat heart: the role of G-proteins and adrenergic stimulation. Mol Cell Biochem 147: 123-128, 1995.

SHEN D, DALTON TP, NEBERT DW, SHERTZER HG: Glutathione redox state regulates mitochondrial reactive oxygen production. $J$ Biol Chem 280: 25305-25312, 2005.

STOWE DF, CAMARA AK: Mitochondrial reactive oxygen species production in excitable cells: modulators of mitochondrial and cell function. Antioxid Redox Signal 11: 1373-1414, 2009.

TILTON RG, KAWAMURA T, CHANG KC, IDO Y, BJERCKE RJ, STEPHAN CC, BROCK TA, WILLIAMSON JR: Vascular dysfunction induced by elevated glucose levels in rats is mediated by vascular endothelial growth factor. J Clin Invest 99: 2192-2202, 1997.

VARADY KA, HUDAK CS, HELLERSTEIN MK: Modified alternate-day fasting and cardioprotection: relation to adipose tissue dynamics and dietary fat intake. Metabolism 58: 803-811, 2009. 
WALKER MJ, CURTIS MJ, HEARSE DJ, CAMPBELL RW, JANSE MJ, YELLON DM, COBBE SM, COKER SJ, HARNESS JB, HARRON DW, ET AL.: The Lambeth Conventions: guidelines for the study of arrhythmias in ischaemia infarction, and reperfusion. Cardiovasc Res 22: 447-455, 1988.

WAN R, CAMANDOLA S, MATTSON MP: Intermittent food deprivation improves cardiovascular and neuroendocrine responses to stress in rats. J Nutr 133: 1921-1929, 2003.

WILLIAMSON DH, LUND P, KREBS HA: The redox state of free nicotinamide-adenine dinucleotide in the cytoplasm and mitochondria of rat liver. Biochem J 103: 514-527, 1967.

WILLIAMSON JR, CHANG K, FRANGOS M, HASAN KS, IDO Y, KAWAMURA T, NYENGAARD JR, VAN DEN ENDEN M, KILO C, TILTON RG: Hyperglycemic pseudohypoxia and diabetic complications. Diabetes 42: 801-813, 1993.

WOLIN MS: Reactive oxygen species and the control of vascular function. Am J Physiol Heart Circ Physiol 296: H539-H549, 2009.

YELLON DM, HAUSENLOY DJ: Myocardial reperfusion injury. N Engl J Med 357: 1121-1135, 2007.

ZHANG X, MIN X, LI C, BENJAMIN IJ, QIAN B, ZHANG X, DING Z, GAO X, YAO Y, MA Y, CHENG Y, LIU L: Involvement of reductive stress in the cardiomyopathy in transgenic mice with cardiac-specific overexpression of heat shock protein 27. Hypertension 55: 1412-1417, 2010.

ZOU Z, SASAGURI S, RAJESH KG, SUZUKI R: dl-3-Hydroxybutyrate administration prevents myocardial damage after coronary occlusion in rat hearts. Am J Physiol Heart Circ Physiol 283: H1968-H1974, 2002.

ZWEIER JL, TALUKDER MA: The role of oxidants and free radicals in reperfusion injury. Cardiovasc Res 70: 181190, 2006. 